\title{
Chloroquine and hydroxychloroquine as antimalarials and antivirals against SARS-CoV-2: The hemin factor
}

\author{
Tomás Herraiz \\ Instituto de Ciencia y Tecnología de Alimentos y Nutrición (ICTAN). \\ Spanish National Research Council (CSIC). \\ Juan de la Cierva 3, 28006, Madrid (Spain). \\ E-mail: therraiz@ictan.csic.es
}

\begin{abstract}
Drugs are urgently needed for COVID-19. In that context, the antimalarial drugs chloroquine and hydroxychloroquine are being used based on their antiviral action against SARS-CoV-2 in vitro and results from early clinical studies. The antiviral action of these drugs relays on their accumulation into lysosomes and acidic endosomes that increase $\mathrm{pH}$, making more difficult virus entry and replication. In malaria, these drugs accumulate into the Plasmodium acidic vacuole, and interact with hemin released from hemoglobin, inhibiting its crystallization to hemozoin ( $\beta$-hematin). Hemin is toxic for the parasite (antimalarial effect). Recent results have shown that chloroquine inhibits $\beta$-hematin formation, and increases soluble free hemin that is involved in oxidative reactions and inhibition of cysteine proteases, underlining the chloroquine effects mediated by hemin. Therefore, antimalarial and antiviral actions of these drugs differ by the hemin factor. However, could hemin play a role in the antiviral action? Infections by SARS-CoV-2 and acute respiratory distress syndrome (ARDS) induce local lung hemorrhages releasing cell-free hemoglobin which is oxidized and releases free hemin. Besides, SARS-CoV-2 invades epithelial cells of the lung, which express non-erythroid hemoglobin. Chloroquine/hydroxychloroquine could hypothetically interact with that hemin/hemoglobin affecting the host and virus. It could result in oxidative reactions and inhibition of cysteine proteases necessary for viral progression. Remarkably, hemin also exerts antiviral actions against a number of viruses, supporting a possible role in the antiviral effects. Hemin is commonly used for the treatment of acute porphyria attacks, and this might be relevant in possible cases of acute porphyria attacks and COVID-19 treated with antimalarial drugs. Finally, it could be worth of testing the antiviral action of chloroquine/hydroxychloroquine and hemin combined, and that of hemin alone against SARS-CoV-2 (COVID-19). ${ }^{1}$
\end{abstract}

Key words: SARS-CoV-2, COVID-19, Plasmodium, malaria, chloroquine, hydroxychloroquine, hemin, hemoglobin, cysteine proteases, oxidative reactions, antiviral, antimalarial, acute porphyria

\footnotetext{
${ }^{1}$ The information included in this preprint is for scientific use only. The drugs mentioned cannot be taken or used without the legal prescription by a doctor.
} 


\section{Background and SARS-CoV-2 pathogenesis}

In December 2019, the novel coronavirus SARS-CoV-2 was transmitted from person to person leading to a pneumonia disease called coronavirus disease-19 (COVID-19). The WHO has declared COVID-19 a pandemic. SARS-CoV-2 causes symptoms ranging from mild disease to severe lung injury and multi-organ failure, eventually leading to death. Many people have no symptoms or mild symptoms but some develop severe respiratory illness including acute respiratory distress syndrome (ARDS). The new virus is related to previous Severe Acute Respiratory Syndrome Coronavirus (SARS-CoV) that emerged in 2002 and the Middle East Respiratory Syndrome Coronavirus (MERS-CoV) in 2012.

The novel SARS-CoV-2 is an enveloped virus containing one positive-strand RNA genome. It encodes several structural proteins, including the glycosylated spike (S) protein that mediates host cell invasion via binding to a receptor located on the surface of cell membrane. The viral genome encodes non-structural proteins including the RNAdependent RNA polymerase (RdRp), coronavirus main protease (3CLpro), and papainlike protease (PLpro). Upon entrance to the host cells, the viral genome is released and translated into viral polyproteins using host cell protein translation machinery, which are then cleaved into effector proteins by viral proteases 3CLpro and PLpro. RdRp synthesizes a full-length negative-strand RNA template to be used by RdRp to make more viral genomic RNA (Liu et al., 2020). Proteases and RdRp are critical for viral infection and replication and are key targets for drug discovery. SARS-CoV-2 invades human cells through the angiotensin converting enzyme 2 (ACE2) receptor (Zhang et al., 2020; Zhou et al., 2020) that is expressed in the lung, particularly in type II alveolar cells (ATII), but also in extrapulmonary tissues including heart, kidneys, blood vessels, and intestine (Zhang et al., 2020). This wide distribution may explain the multi-organ dysfunction observed in COVID-19 patients. To enter into the cells, SARS-CoV-2 spike (S) protein binds to ACE2 receptors (Walls et al., 2020; Wan et al., 2020; Zhang et al., 2020), and the process requires priming $\mathrm{S}$ protein by transmembrane protease serine 2 (TMPRSS2) of the host cells that cleaves S protein (Hoffmann et al. 2020). In addition, other coreceptors or auxiliary proteases, and mechanisms by which viruses enter cells could be also involved (Lukassen et al. 2020; Hamming et al., 2004). Coronaviruses can enter into the host cell via endosomal and non-endosomal entry in presence of proteases, but SARS$\mathrm{CoV}$ replication primarily depends on the non-endosomal pathway dependent on proteases (Lukassen et al., 2020). Endosomal cysteine proteases of the host (e.g. cathepsins) could also participate in priming of $\mathrm{S}$ protein facilitating virus entry (Hoffmann et al., 2020).

The primary sites of gas exchange with the blood in the alveolus are composed of a thin epithelium that covers most of the surface area and contains alveolar type I cells (ATI) and type II cells (ATII). ATII cells produce the pulmonary surfactant, a fluid coating that facilitates the transfer of gases between the blood and alveolar air. ATII cells are capable of cellular division giving rise to ATI and ATII cells when the lung tissue is damaged. Because of the high expression of ACE2 in ATII cells, these cells are more susceptible to infections by SARS-CoV-2 (Lukassen et al., 2020). Within the alveoli, the oxygen travels through a thin wall from air sacs to small blood vessels (capillaries) that deliver oxygen to red blood cells. SARS-CoV-2 damages the wall and the lining cells of 
alveoli and capillaries making difficult the transfer of oxygen and producing severe respiratory illness.

Currently, there is no approved treatment for COVID-19. Researchers worldwide are trying to find effective drugs (Guy et al. 2020; Liu et al., 2020). Drugs against other virus such as the protease inhibitors, lopinavir and ritonavir (HIV) or the virus replication inhibitor, redemsivir (Ebola), are being used in hospitals (Zhang and Liu, 2020). Clinical trials with these drugs are underway. At the same time, old drugs are being considered and repurposed for COVID-19 (Colson et al. 2020; Guo, 2020; Guy et al. 2020). Chloroquine and hydroxychloroquine (Figure 1) are two oral antimalarial drugs also used to treat rheumatoid arthritis and autoimmune diseases like lupus. These drugs have reached a remarkable attention in COVID-19 after promising results obtained by Chinese and French researchers that have generated high expectations although not exempt of scepticism for many. As a result, they are actually employed to treat COVID-19 patients in hospitals. A number of clinical trials are underway to determine their efficacy against SARS-CoV-2 as well as their ability to modulate the immune system and mitigate inflammation (the so-called cytokine storm) that ultimately may result in organ failure. These drugs reduce inflammatory cytokines IL-1, IL-6, TNF $\alpha$ produced by various cell types and IFN $\gamma$ by mononuclear cells (Savarino et al., 2003; Schrezenmeier and Dörner, 2020). They get inside acidic vesicles such as the lysosomal compartment, affect lysosomal activity and autophagy, and inhibit signalling pathways (Schrezenmeier and Dörner, 2020). The inflammatory attack could be somehow reduced by their accumulation in lymphocytes and macrophages. The pharmacology of chloroquine and hydroxychloroquine is similar. They are usually well tolerated and their half-lives are long (40-60 days), explaining the long duration of effects (Schrezenmeier and Dörner, 2020). Following, the antiviral and antimalarial actions of chloroquine and hydroxychloroquine are reviewed and discussed with a focus on the mechanisms of action, and subsequently a possible role for hemin/hemoglobin is considered in SARSCoV-2 infection (COVID-19).

\section{Chloroquine and hydroxychloroquine as antivirals against SARS-CoV-2}

Chloroquine (and hydroxychloroquine) has a broad spectrum of antiviral activity against HIV, hepatitis B, Herpex simplex virus type 1, Ebola and also SARS and MERS (Devaux et al., 2020; Savarino et al., 2003). It exerts antiviral effects by inhibiting pH-dependent steps of replication of flaviviruses, retroviruses, and coronaviruses. Savarino et al (2003) already suggested that chloroquine could be used in the future in SARS diseases. Its antiviral action pre- and post-infection against SARS and other coronavirus has been demonstrated in in vitro studies (Keyaerts et al., 2004 and 2009; Vincent et al., 2005). Chloroquine affected early stages of SARS coronavirus replication. The effective concentration $\left(\mathrm{EC}_{50}=8.8 \mu \mathrm{M}\right)$ for the inhibition of SARS-CoV in vitro was significantly lower than its cytostatic activity (selectivity index (SI) of 30), and the $\mathrm{EC}_{50}$ approximates the plasma concentration of chloroquine during treatment of acute malaria (Keyaerts et al., 2004). Chloroquine showed strong in vitro and in vivo antiviral activities against human coronavirus HCoVOC43 (common cold), and treatment with daily doses of chloroquine had a long-lasting protective effect against lethal coronavirus OC43 infection 
in newborn mice (Keyaerts et al., 2009). The exact mechanism of the antiviral action of chloroquine and hydroxychloroquine is not completely known. Viruses can enter host cells by endocytosis and target to the lysosomal compartment where the low $\mathrm{pH}$ and the action of enzymes disrupt the viral particle liberating nucleic acids and enzymes necessary for viral replication (Figure 2B). Chloroquine and hydroxychloroquine are weak bases that can affect acid vesicles leading to dysfunction of enzymes. They concentrate within acidic organelles such as the endosome, Golgi vesicles and lysosomes, where the $\mathrm{pH}$ is relatively low and these molecules are charged. As a result, the $\mathrm{pH}$ of these organelles increases and disrupts acid hydrolases inhibiting post-translational modification of the newly synthetized proteins and other enzymes involved in the virus replication pathways (Savarino et al., 2003). Chloroquine impairs replication of viruses by interacting with endosome-mediated viral entry and in the late stages of replication (Savarino et al., 2003). However, SARS-CoV-2's main entryway uses the spike S protein to attach to ACE2 receptor on the surface of human cells instead to endosomes (Kupferschmidt and Cohen, 2020) (Figure 2B). For enveloped viruses such as SARS$\mathrm{CoV}-2$, post-translational modification of the enveloped glycoproteins involves proteases and glycosyl transferases, some of which require low $\mathrm{pH}$. Chloroquine could inhibit viral replication by affecting proteolytic processing of those proteins. On the other hand, the anti-SARS effect of chloroquine was also due to the inhibition of glycosylation of the SARS coronavirus receptor ACE2 (Vincent et al., 2005).

Chloroquine inhibits the growth of SARS-CoV-2 in vitro $\left(\mathrm{EC}_{50}=1.13 \mu \mathrm{M}\right.$; $\mathrm{SI}>$ 88.50) (Wang et al., 2020). Hydroxychloroquine also inhibits SARS-CoV-2 in vitro (EC 50 $=0.72 \mu \mathrm{M})$, and is more potent than chloroquine $\left(\mathrm{EC}_{50}=5.47 \mu \mathrm{M}\right)($ Yao et al., 2020). An early clinical study conducted in COVID-19 Chinese patients showed that chloroquine had a significant effect on viral clearance and clinical outcome (reduction in pneumonia severity and time of hospitalization) when compared with control group (Gao et al., 2020). Chinese experts recommended treatment with chloroquine in patients with COVID-19, and started clinical trials (Multicenter collaboration group; Cortegiani et al., 2020). The expectations highly increased with a small clinical study in France suggesting that the treatment of COVID-19 patients with hydroxychloroquine and an antibiotic was useful against the virus (Gautret et al., 2020). Patients received hydroxychloroquine (600 $\mathrm{mg}$ ) daily and their viral load was tested while untreated patients were included as negative controls. The presence and absence of the virus at day- 6 post inclusion was considered the end point. Researchers treated 20 cases and the cases showed a significant reduction of the viral carriage compared with controls. Some patients did not respond to the treatment but it was attributable to metabolic reasons. The antibiotic azithromycin added to hydroxychloroquine significantly improved virus elimination. The researchers concluded that the treatment with hydroxychloroquine was significantly associated with a viral load reduction/disappearance of virus in COVID-19 patients and its effect was reinforced by azithromycin. As a result, they recommended treatment of patients with hydroxychloroquine and azithromycin to cure infection and to limit the transmission of the virus (Gautret et al., 2020). In a recent small clinical trial, the use of hydroxychloroquine (400 mg/day) significantly shortened the time to clinical recovery and promoted the absorption of pneumonia among patients with COVID-19 (Preprint: Chen et al. 2020). Despite those initial promising results, there is consensus that further randomized clinical trials and additional studies are needed, and some are underway. 
Meanwhile, hydroxychloroquine and chloroquine are currently being administered to hospitalized COVID-19 patients in many countries. Nevertheless, the controversy continues. For example, a recent retrospective non-randomized study with COVID-19 patients showed no benefit of hydroxychloroquine regarding death outcome (Preprint: Magagnoli et al., 2020) whereas in another study increased survival is reported (Preprint: Membrillo et al., 2020). Finally, in a recently reported multinational registry analysis, hydroxychloroquine and chloroquine had no benefit and increased frequency of ventricular arrhythmias (Mehra et al. 2020).<smiles>CCN(CC)CCCC(C)Nc1ccnc2cc(Cl)ccc12</smiles>

chloroquine<smiles>CCN(CCO)CCCC(C)Nc1ccnc2cc(Cl)ccc12</smiles>

hydroxychloroquine

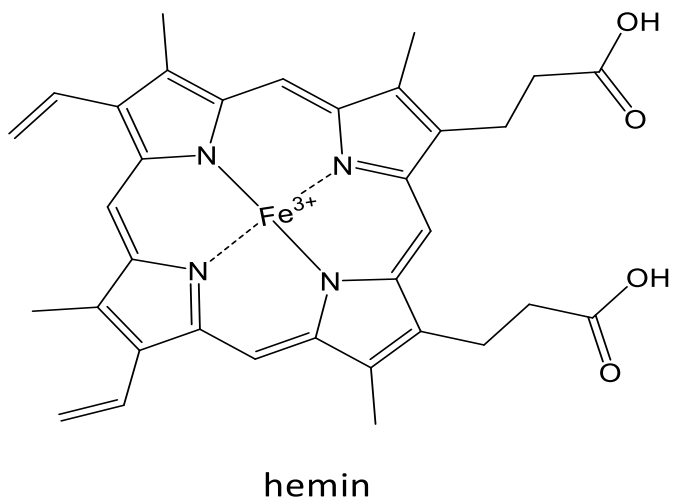

Figure 1. Chemical structures of chloroquine, hydroxychloroquine and hemin. 


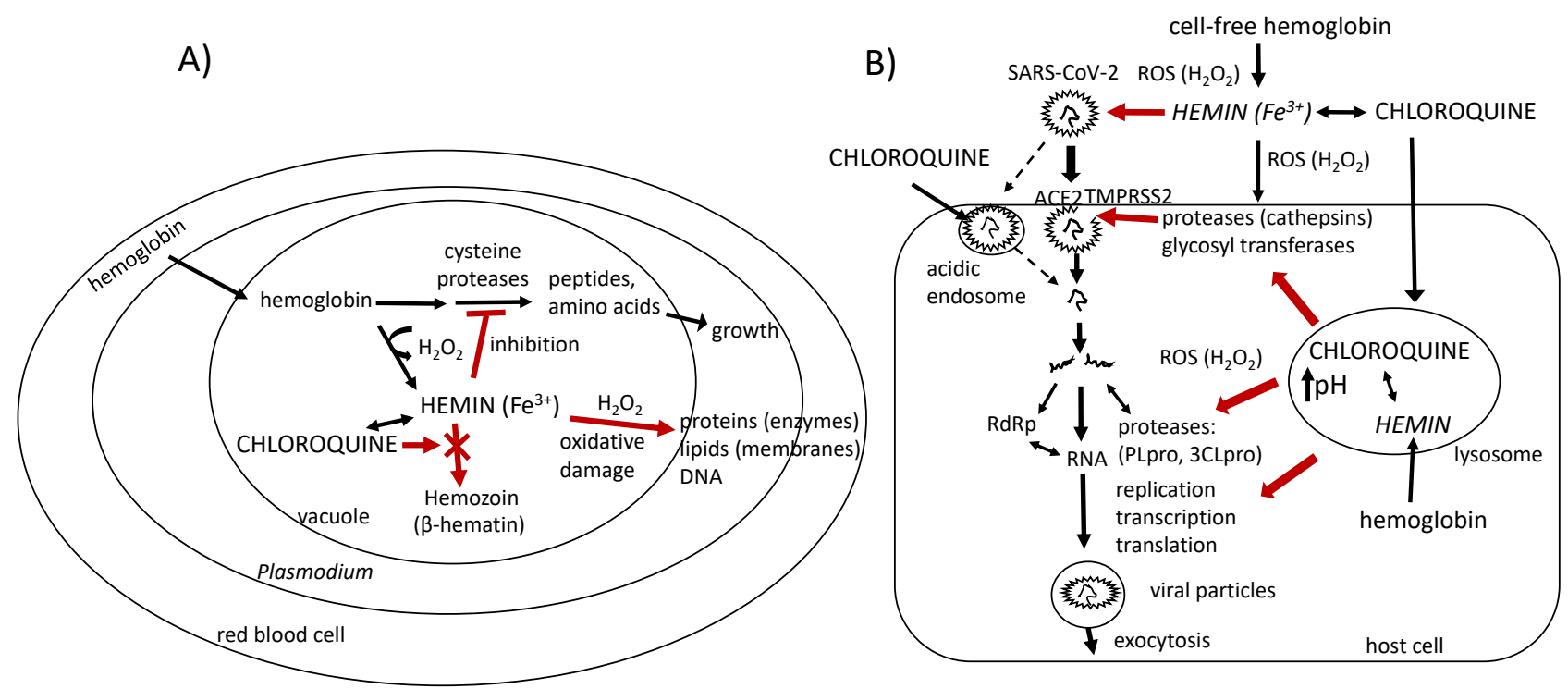

Figure 2. (A) Chloroquine and hydroxychloroquine as antimalarials. Plasmodium breaks down hemoglobin from erythrocytes to amino acids that are used for growth and survival. During this process, hemin $\left(\mathrm{Fe}^{3+}\right)$ and $\mathrm{H}_{2} \mathrm{O}_{2}$ are released. Hemin is toxic but it is converted into the non-toxic pigment hemozoin ( $\beta$-hematin). Chloroquine inhibits this conversion and proportionally increases soluble hemin that is involved in peroxidative reactions (oxidation) and inhibition of cysteine proteases (Herraiz et al., 2019). These mechanisms can cause oxidative damage in proteins (enzymes), membranes and DNA, and inhibit hemoglobin degradation, affecting parasite growth and survival. Hydroxychloroquine works similarly. (B) Chloroquine and hydroxychloroquine as antivirals. Chloroquine accumulates into lysosomes and acidic vesicles (endosomes) of host cell and increases $\mathrm{pH}$, affecting enzymes needed for virus entry and infection such as proteases and glycosyl transferases, and enzymes used for replication (Savarino et al., 2003). Hypothetically, in the presence of cell-free hemoglobin, hemin and $\mathrm{H}_{2} \mathrm{O}_{2}$ (ROS) are released. Hemin could interact with chloroquine and trigger oxidative reactions and inhibition of cysteine proteases that may affect host cell and virus. Non-erythroid hemoglobin could be degraded into lysosomes, and hypothetically release free hemin and ROS that could interact with chloroquine exhibiting oxidative actions and inhibition of cysteine proteases and other enzymes needed for virus replication. Hydroxychloroquine might work similarly. The role of hemin/hemoglobin in the graph B and its interaction with chloroquine is hypothetical and needs to be confirmed. This Figure cannot be reproduced without author permission as it contains information that need to be confirmed in scientific reports.

\section{Antimalarial actions of chloroquine and hydroxychloroquine}

Plasmodium falciparum passes over several stages during its infection cycle in malaria. In its erythrocyte stage, the parasite enters into the red blood cells and digests hemoglobin releasing heme, and peptides and amino acids that are used for growth and survival (Figure 2A). Heme is toxic for the parasite but it has a detoxification system that consists in heme crystallization to afford the insoluble pigment hemozoin (ferriprotoporphyrin IX dimer or $\beta$-hematin) (Kumar et al., 2007). The heme crystallization system of Plasmodium is a target for antimalarial drugs (Egan, 2002; Kumar et al., 2007; Mossadaque et al., 2018). Chloroquine and other quinolines exert antimalarial actions by 
interfering with this system. They accumulate into the acidic digestive vacuole of the parasite (Khun et al., 2007), and prevent heme sequestration resulting in toxicity. The biochemical events underlying the toxic action of heme are poorly understood. In a recent work, we have reported that active quinolines inhibit the formation of $\beta$-hematin and proportionally increase soluble free hemin (ferriprotoporphyrin IX) (Herraiz et al., 2019) (Figure 1). Among the quinolines and $\beta$-carbolines studied, one of the most active was chloroquine. Chloroquine interacts with hemin by forming soluble complexes (Sullivan et al. 1996). We showed that active quinolines increased free hemin that catalyzed peroxidative reactions under conditions occurring in digestive vacuole (Herraiz et al., 2019) (Figure 2A). A significant correlation was found between free hemin increased due to the action of chloroquine and peroxidation (oxidative effects). Then, chloroquine increases oxidative effects in the parasite through increased soluble free hemin. The most active pro-oxidant drugs were chloroquine and quinacrine whereas drugs that did not enhance soluble free hemin such as 8-hydroxyquinoline (a phenolic compound) or $\beta$ carbolines did not promote oxidation.

Increased peroxidation produced by chloroquine depended on hemin and it was evidenced in presence of $\mathrm{H}_{2} \mathrm{O}_{2}$ (Herraiz et al., 2019). Reactive oxygen species (ROS) can participate in the mechanism of action. Plasmodium breaks down hemoglobin to peptides and amino acids, and releases heme $\left(\mathrm{Fe}^{2+}\right)$ which converts into hemin $\left(\mathrm{Fe}^{3+}\right)$. As a result, it generates superoxide anion $\left(\mathrm{O}_{2}{ }^{\bullet-}\right)$ that dismutates to $\mathrm{H}_{2} \mathrm{O}_{2}$ (Egan, 2008) (Figure 2A). $\mathrm{H}_{2} \mathrm{O}_{2}$ could be involved in peroxidase reactions catalyzed by increased levels of free hemin allowed by the chloroquine action (Herraiz et al., 2019). Results also suggested that proteins (globin and others) potentiate peroxidation catalyzed by free hemin. Although chloroquine contributed to oxidative stress by increasing free hemin, it had no pro-oxidant effects by itself. Peroxidative actions depended on soluble free hemin although hemin could be as hemin-chloroquine complex. Then, the accumulation of chloroquine into the digestive vacuole of Plasmodium increases soluble free hemin which can trigger oxidative reactions resulting in oxidative damage to membranes, proteins, and DNA, with the subsequent toxicity for the parasite (Kumar et al., 2005).

Plasmodium uses hemoglobin from red blood cells as a major source of nutrients. Hemoglobin is shuttled to vacuole where it is digested by several proteases, including cysteine proteases such as papain-like falcipains (Goldberg et al., 1990; Rosenthal, 2011). This process results in high levels of globin, peptides, and amino acids that are used for the parasite growth. Cysteine proteases are involved in essential processes for parasite survival and growth such hemoglobin digestion but also erythrocyte rupture and host cell invasion (Lisk et al., 2008; Rosenthal, 2004 and 2011). Therefore, the inhibition of proteolysis is a target for antimalarial drugs (Himangini et al., 2018; Roy, 2017). Recent results indicate that hemin enhanced by the action of chloroquine is able to inhibit different cysteine proteases such as papain, ficin and cathepsin B (Herraiz et al., 2019). Inhibition was due to soluble free hemin (or hemin-chloroquine complexes) allowed by the action of chloroquine but not to chloroquine itself, and occurred in presence of $\mathrm{H}_{2} \mathrm{O}_{2}$ suggesting a likely involvement of oxidative reactions (Figure 2A). The inhibition of cysteine proteases by hemin increased in presence of proteins such as globin and others. These results suggest that chloroquine could interfere with hemoglobin digestion during the blood stages of malarial life cycle and inhibits lysosomal functions and protein degradation. 
Chloroquine and hydroxychloroquine have also antibacterial and antifungal activity (Rolain et al., 2007; Yeo et al., 2020). Hydroxychloroquine is associated with a decreased risk of major fungal infections (Pneumocystis pneumonia) in lupus patients (Yeo et al., 2020). Antibacterial activities are mainly due to $\mathrm{pH}$-dependent iron depletion and to increased $\mathrm{pH}$ of phagolysosomes (acid vesicles), which inhibit the growth of intracellular organisms.

\section{Chloroquine and hydroxychloroquine as antivirals in COVID-19: A role for hemin/hemoglobin}

The results discussed above suggest that chloroquine and hydroxychloroquine work differently against virus and Plasmodium. The main differential factor is the hemin/hemoglobin. Against virus like SARS-CoV-2, they work by diminishing the efficiency of the virus at entering host cells and decreasing its replication rate. In malaria, they work by poisoning the parasite digestive system (vacuole) via increasing free hemin that is toxic through oxidative stress and enzyme inhibition (cysteine proteases and others). The targeted cells are also different: ACE2-expressing cells such as epithelial ATII cells and other extrapulmonary cells in SARS-CoV-2 infection, and erythrocytes in malaria. In both cases, cell systems where chloroquine or hydroxychloroquine accumulate are involved: lysosomes and related acidic compartments of host cells in SARS-CoV-2, and digestive vacuole in malaria parasite. It is thought that chloroquine and hydroxychloroquine exert their antiviral actions mainly via endosomes. However, the main entryway of SARS-CoV-2 is by the spike $\mathrm{S}$ protein instead of endosomes (Figure 2B). This may suggest that additional mechanisms could be involved in their antiviral action.

Most drug candidates investigated in COVID-19 target key proteins of the host cell or the virus. The host's cell proteins are TMPRSS2 protease or cathepsins (used for virus entrance) and enzymes ACE2 (virus receptor). The targeted proteins of the virus are the $\mathrm{S}$ protein (a glycoprotein), the RNA-dependent RNA polymerase (RdRp), and the two proteases that are essential for viral replication: main protease (3Clpro), a chymotrypsinlike cysteine protease, and papain-like cysteine protein (PLpro). The 3CLpro utilizes a Cys/His catalytic dyad whereas PLpro has a classical Asp/His/Cys catalytic triad (Preprint: Stoermer, 2020a,b). PLpro possesses a C-terminal zinc-finger like domain bearing four cysteine residues that are critical for catalytic function (Preprint: Stoermer, 2020b). Drugs are being tested as inhibitors of these cysteine proteases in order to block viral replication.

Chloroquine is not a good inhibitor of cysteine proteases. However, it interacts with hemin and increases oxidative reactions (oxidative stress) and inhibition of cysteine proteases (Herraiz et al., 2019). These events might hypothetically occur in the host and virus during infection. Cell cultures might contain residual hemoglobin (methemoglobin) and hemin eventually influencing the antiviral action of chloroquine or hydroxychloroquine. In vivo, hemoglobin is confined into red blood cells, which in contrast to Plasmodium, are not presumably targets of SARS-CoV-2. Recent results suggest that the virus might use CD147 receptor as an additional gate into host cells and the same receptor is used by Plasmodium to enter into red blood cells (Preprint: Wang et 
al., 2020; Zhang et al., 2018). However, noticeable hemolysis has not been reported as a common feature in COVID-19 patients although ferritin appears to increase. Nevertheless, there are possible sources of extravascular local cell-free hemoglobin and hemin that should be considered. Hemoglobin could be locally released from red blood cells breakdown in capillaries close to alveolar cells damaged during infection. Thus, SARS-CoV infections caused hemorrhages in the alveolar space of the lungs (Gralinski and Baric, 2015), and lung hemorrhages in animal models infected with coronaviruses are significantly reduced with antivirals (Sheahan et al., 2020). SARS-CoV-2 attacks blood vessels and some patients show coagulation dysfunction and develop blood clots while others develop pulmonary embolism. The lung pathology seen in patients with COVID-19 shows marked microvascular thrombosis and hemorrhage linked to extensive alveolar and interstitial inflammation (Preprint: McGonagle et al., 2020). Clinical pathological studies of lung biopsies and autopsies of COVID-19 patients indicate alveolar hemorrhage along with small vessel thrombus formation in the lung periphery (Fox et al., 2020; Preprint: Luo et al., 2020). Alveolar hemorrhage is also a common feature of the acute respiratory distress syndrome (ARDS), a condition associated with COVID-19. ARDS patients have elevated levels of cell-free hemoglobin within the alveolar space from red blood cell breakdown (Janz and Ware, 2015; Shaver et al., 2016), and it may contribute to the pathogenesis of acute lung injury. Cell-free hemoglobin resulted in increased inflammation, loss of alveolar capillary barrier integrity, and epithelial cell injury (Shaver et al., 2016). It converts to methemoglobin and releases free hemin (Schaer et al. 2013a). Hemin increased alveolar-capillary permeability (barrier dysfunction) but did not cause alveolar inflammation or epithelial cell injury in the lungs (Shaver et al., 2016). Finally, the drugs chloroquine and hydroxychloroquine might also induce erythrocyte breakdown in some patients (Chou and Fitch, 1980; Juurlink, 2020). Extracellular hemin/hemoglobin released from red blood cell breakdown might be a target for the action of chloroquine and hydroxychloroquine (Figure 2B).

On the other hand, hemoglobin is expressed in non-erythroid cells such as epithelial cells and activated macrophages. Expression of this protein has been observed in several human tissues including the lungs, particularly in alveolar epithelial cells type II (ATII) that produce the pulmonary surfactant essential for lung function (Bhaskaran et al., 2005; Ghost et al., 2018; Grek et al., 2011; Ishikawa et al., 2010; Newton et al., 2006; Saha et al., 2014). Hypoxia greatly increased hemoglobin expression in ATII cells, and decreased surfactant proteins (Grek et al., 2011). Hemoglobin in these cells might be involved in sensing oxygen concentrations, and facilitation of oxygen transport across air-blood barrier (Grek et al., 2011), and it might have a role in ARDS. Remarkably, epithelial ATII cells that are main targets of SARS-CoV-2 infection also express hemoglobin, and this expression highly increases during hypoxia, a phenomenon occurring during viral infection. Hemoglobin/hemin expressed in ATII cells might be a target for the action of chloroquine and hydroxychloroquine (Figure 2B).

Local extracellular hemoglobin or hemoglobin expressed in ATII cells could be oxidized to methemoglobin $\left(\mathrm{Fe}^{3+}\right)$. This oxidation produces reactive oxygen species (ROS) $\left(\mathrm{H}_{2} \mathrm{O}_{2}\right)$, and may occur in presence of inflammatory-cell-derived oxidants (Balla et al., 1993). Methemoglobin is toxic, generates ROS, and releases free hemin (Bunn and Jandl, 1968) which can be incorporated into endothelial cells (Balla et al., 1993, Vercellotti et al., 1994), and perhaps other cells as well (Schaer et al. 2013a). Endothelium 
defends itself from heme/hemin by induction of the heme degrading enzyme heme oxygenase, and produces large amounts of the iron binding protein ferritin (Vercellotti et al., 1994). Chloroquine and hydroxychloroquine could modulate those actions. For example, their interaction with free hemin could trigger oxidative reactions and inhibition of cysteine proteases of the virus and host, which could result in antiviral actions. Chloroquine could also limit degradation of extracellular hemoglobin by alveolar macrophages. During disposition of extracellular hemoglobin in hemoglobin excavenger receptor CD163-positive HEK cells, chloroquine action resulted in intracellular hemoglobin trapping compromising clearance and abolished expression of heme oxygenase-1 (HO-1) (Schaer et al., 2013b). Chloroquine blocked the elimination of hemoglobin (or methemoglobin) by interfering with lysosomal hemoglobin degradation, and caused intracellular accumulation of heme and globin (Schaer et al., 2013b). These results are in line with chloroquine effects by increasing free hemin (and hemoglobin) to potentiate oxidative reactions (oxidative stress) and inhibition of cysteine proteases (Herraiz et al., 2019) that might affect the host and virus (Figure 2B). However, in order to be effective, this mechanism would need the presence of sufficient local free hemin/hemoglobin to interact with chloroquine. It is currently unknown whether this really occurs during infection in COVID-19. In a recent preprint by Liu and Li (2020), it is reported that non-structural proteins of SARS-CoV-2 could attack hemoglobin and heme to release porphyrins used for the virus. This might be interpreted that the virus degraded hemoglobin. However, the report is a bioinformatics study and not an experimental work, and does not prove the breakdown of hemoglobin or heme to porphyrins, that would need a kind of heme oxygenase.

As described here, chloroquine and hydroxychloroquine exert diverse biological effects as shown also by their anti-inflammatory actions in autoimmune (lupus) and rheumatoid arthritis diseases (Ponticelli et al., 2017). They are also used in porphyria such as porphyria cutanea tarda (Singal et al., 2012). As discussed here, an interaction of these drugs with heme is expected. In this regard, intravenous hemin (hydroxyhemin or hemin arginate) is used for the treatment of acute porphyria attacks. It helps to overcome the relative deficiency of heme in the liver and reduces the supply of porphyrins and precursors needed for heme production. During the current COVID-19 pandemic, porphyria patients could be infected with SARS-CoV-2 and still be treated with hemin (https://porphyriafoundation.org/for-patients/COVID-19-and-porphyria/). Harmful drugs are avoided during treatment with hemin although hydroxychloroquine or chloroquine might be probably safe based on evidence that is either inconsistent or insufficient to be conclusive (https://porphyriafoundation.org/drugdatabase/drug-safety-database-search/). So far, it is unknown whether possible simultaneous cases of acute porphyria attack and COVID-19 have been treated with hemin and chloroquine or hydroxychloroquine but it would be of interest to know the outcome because an interaction of hemin with these drugs is expected.

Remarkably, hemin exhibits antiviral actions against several viruses such as HIV, Zika, influenza, or Ebola. This action could be related with the induction of heme oxygenase enzyme. Therefore, the antiviral action of hemin supports a possible mechanism for antiviral action of chloroquine and hydroxychloroquine with involvement of hemin, as discussed above. Hemin inhibited HIV replication in vitro and reduced viral load in mice (Devadas and Dhawan, 2006). It highly inhibited replication of Zika virus in vitro (Huang et al., 2016). Hemin showed anti-influenza virus activity in vitro and in vivo 
(Wang et al., 2017). It ameliorated influenza pneumonia and attenuated virus-induced lung injury, lymphocytopenia and local inflammation in a mouse model. Hemin protected mice from death and body weight loss caused by influenza A virus infection (Wang et al., 2017). Hemin had an important effect in the reduction of Ebola virus replication. Treatment of different human cells with hemin reduced infection by $>90 \%$ and showed minimal toxicity to infected cells (Huang et al., 2016). This inhibition could be partly due to heme oxygenase (OH-1) activity and expression. The reverse transcriptase activity of a murine leukemia virus was inhibited by hemin and inhibition increased 1000-times when hemin solution was aged (Tsutsui et al., 1987). Hemin also potentiated the activity of artemisinin against Hepatitis C virus showing synergistic effects (Paeshuyse et al., 2006). Taken together, the antiviral effects of hemin are noticeable and could be useful in the current context of absence of effective treatments in COVID-19. In this regard, the action of hemin, and the combined action of hemin and chloroquine or hydroxychloroquine may be worth of testing in SARS-CoV-2 (COVID-19) studies evaluating antiviral action and effects on the disease. Nevertheless, possible safety and toxic effects should be also considered as hemin may induce toxic actions (Van Avondt et al., 2019), and chloroquine and hydroxychloroquine are not exempt of undesirable effects such as hearth problems and retinopathy (Chorin et al., 2020; Juurlink, 2020).

\section{Overview and future directions}

In COVID-19, SARS-CoV-2 infects lung epithelial ATII cells and damages the alveolar wall making difficult oxygen exchange. To enter into the cells, SARS-CoV-2 uses spike (S) protein that binds to ACE2 receptors in host cells, and is primed by membrane protease TMPRSS2 and other proteases (cathepsins) of the host. The virus expresses two key proteases, the main protease (3CLpro) and the papain-like protease (PLpro), and a polymerase (RpRp), which are needed for replication. Drugs against SARS-CoV-2 are urgently needed and a number of molecules are being tested and evaluated in clinical trials. Chloroquine and hydroxychloroquine have shown broad antiviral effects in vitro against different viruses including SARS-CoV-2. Early clinical results suggest that these drugs could be useful in COVID-19 patients and on this basis, they are currently being used in hospitals. However, these drugs are not yet approved for COVID-19 and clinical trials are underway. Their antiviral action is mainly attributed to an increase of $\mathrm{pH}$ in lysosomes and acidic vesicles of the host cell that makes difficult the entrance of the virus, and also affects enzymes needed for virus progression. Chloroquine and hydroxychloroquine are approved as antimalarials and in autoimmune diseases. They exert anti-inflammatory effects that could be useful against inflammatory developments occurring in COVID-19. As antimalarials, they get inside the parasite vacuole in infected red blood cells, and inhibit hemin crystallization to hemozoin ( $\beta$-hematin). Soluble hemin is toxic for the parasite. Recent findings have shown that chloroquine inhibits the formation of $\beta$-hematin and increases soluble free hemin that is involved in peroxidative reactions (oxidative stress) and inhibition of cysteine proteases. These mechanisms can underline the toxic effects of chloroquine against the parasite. 
The mechanisms of action of chloroquine and hydroxychloroquine as antivirals and antimalarials differ. The main differential factor is the hemin/hemoglobin. However, local hemorrhages occur in COVID-19 and ARDS patients with a subsequent release of cellfree hemoglobin (extracellular) which is oxidized to methemoglobin and releases hemin. On the other hand, lung epithelial ATII cells invaded by SARS-CoV-2 express nonerythrocytic hemoglobin, which is highly increased during hypoxia. In the presence of sufficient hemoglobin/hemin, chloroquine and hydroxychloroquine could have antiviral effects mediated by hemin. These drugs could interact with hemin and increase oxidative actions and inhibition of cysteine proteases of the host and virus. These processes could affect the virus and host with potential effects on the disease. In the short future, the efficacy and safety of chloroquine and hydroxychloroquine should be conveniently substantiated in clinical trials of COVID-19. Moreover, studies could investigate whether their mechanism of action against SARS-CoV-2 involves oxidative reactions (ROS) and inhibition of cysteine proteases of the virus and host, and the role of hemin/hemoglobin. Remarkably, hemin exerts antiviral actions against a number of viruses both in vitro and in vivo. This fact supports a role of hemin in the antiviral action of chloroquine and hydroxychloroquine. Hemin is used for the treatment of acute porphyria attacks. In this regard, it would be of interest to know whether possible simultaneous cases of porphyria attacks and COVID-19 have been treated with hemin and chloroquine/hydroxychloroquine, and to know the outcome because an interaction among these drugs is expected. Under current need of drugs, it could be worth of testing the antiviral action and safety of chloroquine/hydroxychloroquine combined with hemin and that of hemin alone against SARS-CoV-2 (COVID-19). ${ }^{1}$

\section{References}

Balla, J., Jacob, H.S, Balla, G., Nath, K., Eaton, J.W., Vercelotti, G.M. Endothelial-cell heme uptake from heme proteins: Induction of sensitization and desensitization to oxidant damage. Proc. Natl. Acad. Sci. USA 90, 9285-9289 (1993).

Bhaskaran M., Chen H., Chen Z., Liu L. Hemoglobin is expressed in alveolar epithelial type II cells. Biochem. Biophys. Res. Co. 333, 1348-1352 (2005). https://doi.org/10.1016/j.bbrc.2005.06.042

Bunn, H. F., Jandl, J. H. Exchange of heme among hemoglobins and between hemoglobin and albumin. J. Biol. Chem. 243, 465-475 (1968).

Chen, Z, Hu, J., Zhang, Z., Jiang, S., Han, S., Yan, D., et al. Efficacy of hydroxychloroquine in patients with COVID-19: results of a randomized clinical trial. Preprint medRxiv (2020). doi: https://doi.org/10.1101/2020.03.22.20040758

Chorin, E., Dai, M., Shulman, E., Wadhwani, L., Bar-Cohen, R. et al. The QT interval in patients with COVID-19 treated with hydroxychloroquine and azithromycin. Nat. Med. (2020). https://doi.org/10.1038/s41591-020-0888-2

Chou, A.C., Fitch, C.D. Hemolysis of mouse erythrocytes by ferriprotoporphyrin IX and chloroquine. Chemotherapeutic implications. J. Clin. Invest. 66, 856-858 (1980). https://doi.org/10.1172/JCI109925 
Colson, P., Rolain, J.M., Lagier, J.C., Brouqui, P., Raoult, D. Chloroquine and hydroxychloroquine as available weapons to fight COVID-19. Int. J. Antimicrob. Ag. 55, 105932 (2020). doi: 10.1016/j.ijantimicag.2020.105932

Cortegiani, A., Ingoglia, G., Ippolito, M., Giarratano, A., Einav, S. A systematic review on the efficacy and safety of chloroquine for the treatment of COVID-19. J. Critical Care, 57, 279-283 (2020). https://doi.org/10.1016/j.jcrc.2020.03.005

Devadas, K., Dhawan, S., Hemin activation ameliorates HIV-1 infection via heme oxygenase-1 induction. J. Immunol. 176, 4252-4257 (2006).

Devaux, C.A., Rolain, J.-M., Colson, P., Raoult, D. New insights on the antiviral effects of chloroquine against coronavirus: what to expect for COVID-19? Int. J. Antimicrob. Ag., art. 105938 (2020). https://doi.org/10.1016/j.ijantimicag.2020.105938

Egan, T. J. Physico-chemical aspects of hemozoin (malaria pigment) structure and formation. J. Inorg. Biochem. 91, 19-26 (2002).

Egan, T. J. Haemozoin formation. Mol. Biochem. Parasitol. 157, 127-136 (2008).

Fox, S.E., Akmatbekov, A., Harbert, J.L., Li, G., Brown, J.Q., Vander Heide, R.S. Pulmonary and cardiac pathology in African American patients with COVID-19: an autopsy series from New Orleans. Lancet Respir. Med. (2020). https://doi.org/10.1016/ S2213-2600(20)30243-5

Gao J, Tian, Z, Yang, X. Breakthrough: Chloroquine phosphate has shown apparent efficacy in treatment of COVID-19 associated pneumonia in clinical studies. Biosci. Trends. 14, 72-73 (2020). doi: 10.5582/bst.2020.01047

Gautret, P., Lagier, J-C., Parola, P., Hoang, V.T., Meddeb, L. et. al. Hydroxychloroquine and azithromycin as a treatment of COVID-19: results of an open-label non-randomized clinical trial. Int. J. Antimicrob. Ag. Article 105949 (2020). doi: 10.1016/j.ijantimicag.2020.105949

Ghosh, A., Garee, G., Sweeny, E.A., Nakamura, Y., Stuehr, D.J. Hsp90 chaperones hemoglobin maturation in erythroid and nonerythroid cells. Proc. Natl. Acad. Sci. USA 115, E1117-E1126 (2018). https://doi.org/10.1073/pnas.1717993115

Goldberg, D. E., Slater, A. F. G., Cerami, A., Henderson, G. B. Hemoglobin degradation in the malaria parasite Plasmodium falciparium: an ordered process in a unique organelle. Proc. Natl. Acad. Sci. USA 87, 2931-2935 (1990).

Gralinski, L. E., Baric R. S. Molecular pathology of emerging coronavirus infections. J. Pathol. 235, 185-195 (2015).

Grek C., Newton D., Spyropoulos D., Baatz J. Hypoxia up-regulates expression of hemoglobin in alveolar epithelial cells. Am. J. Respir. Cell. Mol. Biol. 44, 439-447 (2011). https://doi.org/10.1165/rcmb.2009-0307OC

Guo, D. Old weapon for new enemy: drug repurposing for treatment of newly emerging viral diseases. Virologica Sinica. (2020). https://doi.org/10.1007/s12250-020-00204-7 
Guy, R.K., DiPaola, R.S., Romanelli, F., Dutch, R.E. Rapid repurposing of drugs for COVID-19. Science. 368, 829-830 (2020). doi: 10.1126/science.abb9332

Hamming I., Timens W., Bulthuis M.L, Lely A.T, Navis G., van Goor H. Tissue distribution of ACE2 protein, the functional receptor for SARS coronavirus. A first step in understanding SARS pathogenesis. J. Pathol. 203, 631- 637 (2004).

Herraiz, T., Guillén, H., González-Peña, D., Arán, V.J. Antimalarial quinoline drugs inhibit $\beta$-hematin and increase free hemin catalyzing peroxidative reactions and inhibition of cysteine proteases. Sci. Rep. 9, 15398 (2019). https://doi.org/10.1038/s41598-01951604-z

Himangini, Pathak, D. P., Sharma, V. \& Kumar, S. Designing novel inhibitors against falcipain-2 of Plasmodium falciparum. Bioorg. Med. Chem. Lett. 28, 1566-1569 (2018).

Hoffmann, M.; Kleine-Weber, H.; Schroeder, S.; Kruger, N.; Herrler, T. et al. SARSCoV-2 cell entry depends on ACE2 and TMPRSS2 and is blocked by a clinically proven protease inhibitor. Cell 181, 271-280.e8 (2020). https://doi.org/10.1016/j.cell.2020.02.052

Huang, H., Falgout, B., Takeda, K., Yamada, K.M., Dhawan, S. Nrf2-dependent induction of innate host defense via heme oxygenase-1 inhibits Zika virus replication. Virology 503, 1-5 (2017). https://doi.org/10.1016/j.virol.2016.12.019

Huang, H., Konduru, K., Solovena, V., Zhou, Z.H., Kumari, N. et al. Therapeutic potential of the heme oxygenase-1 inducer hemin against Ebola virus infection. Curr. Trends Immunol. 17, 117-123 (2016).

Ishikawa N., Ohlmeier S., Salmenkivi K., Myllärniemi, M., Rahman, I. et al. Hemoglobin $\alpha$ and $\beta$ are ubiquitous in the human lung, decline in idiopathic pulmonary fibrosis but not in COPD. Respir. Res. 11, 123 (2010). https://doi.org/10.1186/14659921-11-123

Janz, D.R., Ware, L.B. The role of red blood cells and cell-free hemoglobin in the pathogenesis of ARDS. J. Intensive Care 3, 20 (2015). https://doi.org/10.1186/s40560015-0086-3

Juurlink, D.N. Safety considerations with chloroquine, hydroxychloroquine and azithromycin in the management of SARS-CoV-2 infection. CMAJ 192, E450-E453 (2020). https://doi.org/10.1503/cmaj.200528

Keyaerts E, Vijgen L, Maes P, Neyts J, Van Ranst, M. In vitro inhibition of severe acute respiratory syndrome coronavirus by chloroquine. Biochem. Biophys. Res. Co. 323, 264-68 (2004).

Keyaerts, E., Li, S., Vijgen, L., Rysman, E., Verbeeck, J. et al. Antiviral activity of chloroquine against human coronavirus OC43 Infection in newborn mice. Antimicrob. Agents Ch. 53, 3416-3421 (2009). doi:10.1128/AAC.01509-08

Kuhn, Y., Rohrbach, P., Lanzer, M. Quantitative $\mathrm{pH}$ measurements in Plasmodium falciparum-infected erythrocytes using pHluorin. Cell. Microbiol. 9, 1004-1013 (2007). 
Kumar, S., Bandyopadhyay, U. Free heme toxicity and its detoxification systems in human. Toxicol. Lett. 157, 175-188 (2005).

Kumar, S., Guha, M., Choubey, V., Maity, P., Bandyopadhyay, U. Antimalarial drugs inhibiting hemozoin ( $\beta$-hematin) formation: A mechanistic update. Life Sci. 80, 813-828 (2007).

Kupferschmidt, K., Cohen J. Race to find COVID-19 treatments accelerates. Science 367, 1412-1413 (2020). doi: 10.1126/science.367.6485.1412

Lisk, G., Pain, M., Gluzman, I.Y., Kambhampati, S., Furuya, T. et al. Changes in the plasmodial surface anion channel reduce leupeptin uptake and can confer drug resistance in Plasmodium falciparum-infected erythrocytes. Antimicrob. Agents Ch. 52, 2346-2354 (2008). doi:10.1128/AAC.00057-08

Liu, W., Li, H. COVID-19: Attacks the 1-Beta chain of hemoglobin and captures the porphyrin to inhibit human heme metabolism. ChemRxiv. Preprint. (2020): https://doi.org/10.26434/chemrxiv.11938173.v4

Liu, C., Zhou, Q., Li, Y., Garner, L.V., Watkins, S.P. et al. Research and development on therapeutic agents and vaccines for COVID-19 and related human coronavirus diseases. ACS Central Science, 6, 315-331 (2020). doi: 10.1021/acscentsci.0c00272

Luo, W.; Yu, H.; Gou, J.; Li, X.; Sun, Y. et al. Clinical pathology of critical patient with novel coronavirus pneumonia (COVID-19). Preprints 2020, 2020020407

Lukassen, S., Chua, R. L., Trefzer, T., Kahn, N.C., Schneider, M.A. et al. SARS-CoV-2 receptor ACE2 and TMPRSS2 are primarily expressed in bronchial transient secretory cells (2020) The EMBO Journal e105114 (2020). https://doi.org/10.15252/embj.20105114

Magagnoli, J. Narendran, S. Pereira, F., Cummings, T., Hardin, J.W. et al. Outcomes of hydroxychloroquine usage in United States veterans hospitalized with COVID-19. Preprint medRxiv (2020). https://doi.org/10.1101/2020.04.16.20065920

McGonagle, D. O’Donnell, J.S., Sharif, K., Emery, P., Bridgewood, C. Immune mechanisms of pulmonary intravascular coagulopathy in COVID-19 pneumonia. The Lancet Rheumatol. (2020). https://doi.org/10.1016/S2665-9913(20)30121-1

Mehra, M.R., Desai, S.S., Ruschitzka, F., Patel, A.N. Hydroxychloroquine or chloroquine with or without a macrolide for treatment of COVID-19: a multinational registry analysis. The Lancet (2020). doi: https://doi.org/10.1016/S0140-6736(20)311806

Membrillo, F.J.; Ramírez-Olivencia, G.; Estébanez, M.; de Dios, B.; Herrero, M.D. et al. Early Hydroxychloroquine is associated with an increase of survival in COVID-19 Patients: An observational study. Preprints 2020, 2020050057. doi: 10.20944/preprints202005.0057.v2 
Mosaddeque, F., Mizukami, S. Kamel, M. G, Teklemichael, A.A., Van Dat, T. et al. Prediction model for antimalarial activities of hemozoin inhibitors by using physicochemical properties. Antimicrob. Agents Ch. 62, e02424-17, (2018). https://doi.org/10.1128/aac.02424-17

Multicenter collaboration group of Department of Science and Technology of Guangdong Province and Health Commission of Guangdong Province for chloroquine in the treatment of novel coronavirus pneumonia. Expert consensus on chloroquine phosphate for the treatment of novel coronavirus pneumonia. Zhonghua Jie $\mathrm{He} \mathrm{He} \mathrm{Hu} \mathrm{Xi} \mathrm{Za} \mathrm{Zhi.}$ 2020 Mar 12;43(3):185-188. doi: 10.3760/cma.j.issn.1001-0939.2020.03.009.

Newton D.A., Rao K.M., Dluhy, R.A., Baatz, J.E. Hemoglobin is expressed by alveolar epithelial cells. J. Biol. Chem. 281, 5668-5676 (2006).

Paeshuyse, J., Coelmont, L., Vliegen, I., Van hemel, J., Vandenkerckhove, J. et al. Hemin potentiates the anti-hepatitis $\mathrm{C}$ virus activity of the antimalarial drug artemisinin $\begin{array}{llllll}\text { Biochem. Biophys. Res. } & \text { Co. 348, 139-144 }\end{array}$ https://doi.org/10.1016/j.bbrc.2006.07.014

Ponticelli, C., Moroni G. Hydroxychloroquine in systemic lupus erythematosus (SLE). $\begin{array}{lllll}\text { Expert Opinion Drug } & \text { (2017). }\end{array}$ http://dx.doi.org/10.1080/14740338.2017.1269168

Rolain JM, Colson P, Raoult D. Recycling of chloroquine and its hydroxyl analogue to face bacterial, fungal and viral infections in the 21 st century. Int. J. Antimicrob. Ag. 30, 297-308 (2007).

Rosenthal, P. J. Cysteine proteases of malaria parasites. Int. J. Parasit. 34, 1489-1499 (2004).

Rosenthal, P. J., Falcipains and other cysteine proteases of malaria parasites, in: Cysteine Proteases of Pathogenic Organisms, in Robinson, M. W. and Dalton, J. P., (Eds), Springer-Verlag Berlin, Berlin, pp. 30-48 (2011).

Roy, K. K. Targeting the active sites of malarial proteases for antimalarial drug discovery: approaches, progress and challenges. Int. J. Antimicrob. Ag. 50, 287-302 (2017).

Saha D, Reddy KV, Shrof, A., Ayyar, K., Bashir, T., Reddy, K.W.V. Hemoglobin Expression in Nonerythroid Cells: Novel or Ubiquitous? Int. J. Inflamm. Article ID 803237, (2014). doi:10.1155/2014/803237

Savarino, A., Boelaert, J. R., Cassone, A., Majori, G., Cauda, R. Effects of chloroquine on viral infections: an old drug against today's diseases? Lancet Infect. Dis. 3, 722-727 (2003).

Schaer, D. J., Buehler, P.W., Alayash, A.I., Belcher, J.D., Vercellotti, G.M. Hemolysis and free hemoglobin revisited: exploring hemoglobin and hemin scavengers as a novel class of therapeutic proteins. Blood 121, 1276-1284 (2013a).

https://doi.org/10.1182/blood-2012-11-451229 
Schaer, C. A., Laczko, E., Schoedon, G. Schaer, D. J., Vallelian. F. Chloroquine interference with hemoglobin endocytic trafficking suppresses adaptive heme and iron homeostasis in macrophages: The paradox of an antimalarial agent. Oxid. Med. Cell. Longev., Article ID 870472 (2013b). http://dx.doi.org/10.1155/2013/870472

Schrezenmeier, E. and Dörner, T. Mechanisms of action of hydroxychloroquine and chloroquine: implications for rheumatology. Nat. Rev. Rheumatol. 16, 155-166 (2020).

Shaver, C.M., Upchurch, C.P., Janz, D.R., Grove, B.S., Putz, N.D. et al. Cell-free hemoglobin: a novel mediator of acute lung injury. Am. J. Physiol. Lung Cell. Mol. Physiol. 310, L532-L541 (2016). doi:10.1152/ajplung.00155.2015

Sheahan, T.P., Sims, A. C, Zhou, S., Graham, R.L. Pruijssers A.J., et al. An orally bioavailable broad-spectrum antiviral inhibits SARS-CoV-2 in human airway epithelial cell cultures and multiple coronaviruses in mice. Sci. Transl. Med. 12, 541, eabb5883 (2020). doi: 10.1126/scitranslmed.abb5883

Singal, A. K., Kormos-Hallberg, C., Lee, C., Sadagoparamanujam, V.M., Grady, J.J. et al. Low-dose hydroxychloroquine is as effective as phlebotomy in treatment of patients with porphyria cutanea tarda. Clin. Gastroenterol. Hepatol. 10, 1402-1409 (2012).

Stoermer M.J. Homology models of Wuhan coronavirus 3CLpro protease. ChemRxiv Preprint (2020a). https://doi.org/10.26434/chemrxiv.11637294.v3

Stoermer, M. Homology models of the papain-like protease Plpro from coronavirus 2019nCoV. ChemRxiv Preprint (2020b). https://doi.org/10.26434/chemrxiv.11799705.v1

Sullivan, D. J., Gluzman, I. Y., Russell, D. G. \& Goldberg, D. E. On the molecular mechanism of chloroquine's antimalarial action. Proc. Natl. Acad. Sci. USA 93, 1186511870 (1996).

Tsutsui, K., Mueller, G.C. Hemin inhibits virion-associated reverse transcriptase of murine leukemia virus. Biochem. Biophys. Res. Co. 149, 628-634 (1987). https://doi.org/10.1016/0006-291X(87)90414-1

Van Avondt, K., Nur, E.; Zeerleder, S. Mechanisms of haemolysis-induced kidney injury. Nat Rev Nephrol 15, 671-692 (2019). https://doi.org/10.1038/s41581-019-01810

Vercellotti, G.M., Balla, G., Balla, J., Nath, K, Eaton J.W., Jacob H.S. Heme and the vasculature: an oxidative hazard that induces antioxidant defenses in the endothelium. Artificial Cells, Blood Substitutes, and Biotechnology, 22, 207-213 (1994). doi: 10.3109/10731199409117415

Vincent M. J., Bergeron E., Benjannet S., Erickson, B.R., Rollin, P.E. et al. Chloroquine is a potent inhibitor of SARS coronavirus infection and spread. Virology Journal, 2, 69 (2005). doi:10.1186/1743-422X-2-69

Walls A.C., Park, Y-J, Tortorici, M.A., Wall, A., McGuire, A.T., Veesler, D. Structure, function and antigenicity of the SARS-CoV-2 spike glycoprotein. Cell 180, 281-292 (2020). https://doi.org/10.1016/j.cell.2020.02.058 
Wan Y., Shang, J., Graham, R., Baric, R.S., Li, F. Receptor recognition by novel coronavirus from Wuhan: an analysis based on decade-long structural studies of SARS. J. Virol. 94, e00127-20 (2020). https://doi.org/10.1128/jvi.00127-20

Wang, M., Cao, R., Zhang, L., Yang, X., Liu, J. et al. Remdesivir and chloroquine effectively inhibit the recently emerged novel coronavirus $(2019 \mathrm{nCoV})$ in vitro. Cell Res. 30, 269-271 (2020). https://doi.org/10.1038/s41422-020-0282-0

Wang, K., Chen, W., Zhou, Y-S. Lian, J-Q., Zhang, Z., et al. SARS-CoV-2 invades host cells via a novel route: CD147-spike protein. bioRxiv preprint (2020). https://doi.org/10.1101/2020.03.14.988345

Wang, C., Zhang, Y., Han, L., Guo, L., Zhong, H., Wang, J. Hemin ameliorates influenza neumonia by attenuating lung injury and regulating the immune response. Int. J. Antimicrob. Ag. 49, 45-52 (2017). https://doi.org/10.1016/j.ijantimicag.2016.09.030

Yao, X., Ye, F., Zhang, M., Cui, C., Huang, B. et al. In vitro antiviral activity and projection of optimized dosing design of hydroxychloroquine for the treatment of Severe Acute Respiratory Syndrome Coronavirus 2 (SARS-CoV-2). Clin. Infect. Dis. ciaa237 (2020). https://doi.org/10.1093/cid/ciaa237

Yeo, K., Chen, H., Chen, Y., Lin, C., Chen, D. et al. Hydroxychloroquine may reduce risk of Pneumocystis pneumonia in lupus patients: a Nationwide, population-based casecontrol study. BMC Infect Dis 20, 112 (2020). https://doi.org/10.1186/s12879-020-48261

Zhang, L., Liu, Y. Potential interventions for novel coronavirus in China: A systematic review. J. Med. Virol. 92, 479-490 (2020). https://doi.org/10.1002/jmv.25707

Zhang, H., Penninger, J.M., Li, Y., Zhong, N., Slutsky, A.S. Angiotensin-converting enzyme 2 (ACE2) as a SARS-CoV-2 receptor: molecular mechanisms and potential therapeutic target. Intensive Care Med. 46, 586-590 (2020). https://doi.org/10.1007/s00134-020-05985-9

Zhang, M.-Y-, Zhang, Y., Wu, X.-D., Zhang, K. Lin, P. et al. Disrupting CD147-RAP2 interaction abrogates erythrocyte invasion by Plasmodium falciparum. Blood. 131, 11111121 (2018).

Zhou, P., Yang, X.L, Wang, X.G., Hu, B., Zhang, L. et al. A pneumonia outbreak associated with a new coronavirus of probable bat origin. Nature 579, 270-273 (2020). https://doi.org/10.1038/s41586-020-2012-7

\section{Disclosure of Potential Conflicts of Interest}

There are no conflicts of interest.

Figure 2 of this preprint is for scientific discussion and it cannot be reproduced without author permission because it contains hypothetical information that needs to be confirmed further in scientific reports. 


\section{Acknowledgements}

This critical review and viewpoint originates from results in Herraiz et al. (2019). The author thanks Spanish Government-FEDER through projects RTI2018-093940-B-I00, RTI2018-095544-B-I00 and ICTAN-CSIC. Also, thanks to Fernando Vera and Elena Yuste, recipients of a Garantía Juvenil contract from Comunidad de Madrid (Fondo Social Europeo-Iniciativa de Empleo Juvenil).

${ }^{1}$ The information included in this preprint is for scientific use only. The drugs mentioned cannot be taken or used without the legal prescription by a doctor. 